\title{
Serological based monitoring of a cohort of patients with chronic Chagas disease treated with benznidazole in a highly endemic area of northern Argentina
}

\author{
Leticia L Niborski', Vanina Grippo', Sonia O Lafón1', Gabriela Levitus', Facundo García-Bournissen², \\ Juan C Ramirez ${ }^{1}$, Juan M Burgos ${ }^{1}$, Margarita Bisio', Natalia A Juiz¹, Vilma Ayala ${ }^{3}$, María Coppede ${ }^{3}$, \\ Verónica Herrera ${ }^{3}$, Crescencia López ${ }^{3,+}$, Ana Contreras ${ }^{3}$, Karina A Gómez ${ }^{1}$, Juan C Elean ${ }^{3}$, \\ Hugo D Mujica ${ }^{3,4}$, Alejandro G Schijman ${ }^{1}$, Mariano J Levin ${ }^{1,+}$, Silvia A Longhi ${ }^{1 /+}$
}

\begin{abstract}
${ }^{1}$ Instituto de Investigaciones en Ingeniería Genética y Biología Molecular, Consejo Nacional de Investigaciones Científicas y Tecnológicas, Buenos Aires, Argentina ${ }^{2}$ Hospital de Niños Ricardo Gutiérrez, Servicio de Parasitología y Chagas, Buenos Aires, Argentina

${ }^{3}$ Centro Asistencial Cáritas Diocesana, Añatuya, Santiago del Estero, Argentina ${ }^{4}$ Hospital Zonal Añatuya, Santiago del Estero, Argentina
\end{abstract}

This study aimed to evaluate well-documented diagnostic antigens, named B13, $1 F 8$ and JL7 recombinant proteins, as potential markers of seroconversion in treated chagasic patients. Prospective study, involving 203 patients treated with benznidazole, was conducted from endemic areas of northern Argentina. Follow-up was possible in 107 out of them and blood samples were taken for serology and PCR assays before and 2, 3, 6, 12, 24 and 36 months after treatment initiation. Reactivity against Trypanosoma cruzi lysate and recombinant antigens was measured by ELISA. The rate of decrease of antibody titers showed nonlinear kinetics with an abrupt drop within the first three months after initiation of treatment for all studied antigens, followed by a plateau displaying a low decay until the end of follow-up. At this point, anti-B13, anti-1F8 and anti-JL7 titers were relatively close to the cut-off line, while anti-T. cruzi antibodies still remained positive. At baseline, 60.8\% (45/74) of analysed patients tested positive for parasite DNA by PCR and during the follow-up period in 34 out of 45 positive samples $(75.5 \%)$ could not be detected T. cruzi DNA. Our results suggest that these antigens might be useful as early markers for monitoring antiparasitic treatment in chronic Chagas disease.

Key words: Trypanosoma cruzi - chronic Chagas disease - benznidazole treatment - serological follow-up - adverse effects

Chagas disease or American trypanosomiasis, caused by the parasite Trypanosoma cruzi, is endemic in much of the Americas, from the southern United States to Argentina and Chile. It is the fourth most common tropical disease, after malaria, tuberculosis and schistosomiasis, currently affecting 7-6 million people (WHO 2015). The risk of infection with $T$. cruzi is directly related to poverty, but due to migrations, several cases have been reported throughout the world (Lescure et al. 2008, Muñoz et al. 2009, Jackson et al. 2010).

In Argentina, it is estimated as many as 1.5 million patients have Chagas disease and 2.2 million people in risk of T. cruzi infection (WHO 2015). The endemic area covers the north of the country where the conditions, such as high levels of poverty and social exclusion, low population density, mostly rural, subsistence economy, and a weak health system, favor not only T. cruzi infection but also for the development of this disease.

Once the individual acquires the parasite, the infection starts with an acute phase, followed by a chronic stage

doi: 10.1590/0074-02760160006

Financial support: Fundación Bunge and Born, CONICET.

$\dagger$ In memoriam

+ Corresponding author: longhi@dna.uba.ar

Received 11 January 2016

Accepted 15 April 2016 which includes asymptomatic and symptomatic cases, with cardiac, digestive manifestations or mixed patterns (WHO 2015). Up to now, the available treatment is based on two drugs: nifurtimox and benznidazole (BNZ). Chemotherapy against $T$. cruzi infection is strongly recommended for all cases during the acute stage, in children under 15 years old and reactivated infections in immunocompromised patients (Bianchi et al. 2015), but its effectiveness during the chronic stages is still under revision (Cançado 2002, Viotti et al. 2006, 2014). Some studies suggest that BNZ for asymptomatic or early symptomatic cases may improve parasite clearance rates (de Andrade et al. 1996, Sosa-Estani et al. 1998). In 1999, a panel of experts reached the consensus that patients with chronic Chagas disease should be treated with an anti-T. cruzi medication (PAHO 1999). From this recommendation, many studies are being conducted. Thus, results from a multicenter, placebo-controlled trial involving BNZ for the treatment of Chagas cardiomyopathy showed that the drug significantly diminished serum parasite detection, but did not improve cardiac clinical manifestation (Morillo et al. 2015). In parallel, another trial with longterm follow-up in adult patients, is being conducted in Argentina to evaluate whether BNZ treatment change the evolution of chronic Chagas disease (Riarte 2013). Other randomised clinical studies, with shorter follow-up periods, based on the safety and efficacy of new drugs such as posaconazole, studied this drug alone or in combination with BNZ (Molina et al. 2014, and STOP CHAGAS clinical trial, Identifier: NCT01377480). 
After treatment, the criterion of cure in chronic Chagas disease is the persistence of negative parasitological and serological results (Porrás et al. 2015). Unfortunately, T. cruzi lysate antibody seroconversion occurs several years after antiparasitic therapy in most individuals, while parasitological methods are consistently negative (Guedes et al. 2011, Machado-de-Assis et al. 2012). Thus, the identification of early markers of seronegative conversion is an important and imperative step to evaluate Chagas disease treatment.

The aim of this study was to evaluate if well-known serological markers could have an early predictive value and be useful to monitor drug therapy response, by measuring antibody $(\mathrm{Ab})$ levels over time in a cohort of patients with chronic Chagas disease treated with BNZ. For this purpose, we selected the recombinant proteins named B13, 1F8 and JL7, because they are recognised by most chronic chagasic patients and are currently used in commercial kits for diagnosis (Umezawa et al. 1999, 2003, Ponce et al. 2005).

\section{SUBJECTS, MATERIALS AND METHODS}

Patients and study design - Prospective study was carried out from years 2000-2004 in Añatuya, a city located in a highly Chagas disease-endemic area in the Province of Santiago del Estero - Argentina. Three hundred and twelve $T$. cruzi-infected adults volunteers aged 15-55 years were recruited and 270 patients in the asymptomatic or cardiac chronic phase of Chagas disease were eligible.

Inclusion criteria were positive diagnostic for Chagas disease by commercial ELISA and indirect hemagglutination assays, according to Argentina's National Guidelines and the World Health Organization (WHO) recommendations. Exclusion criteria included the visceral damage, presence of systemic arterial hypertension, liver or kidney failure, severe cardiac lesions associated with other cardiac diseases, pregnancy, lactation, alcoholism and hypersensitivity to the drug. BNZ was given three times daily over 60 days at a total dose $5 \mathrm{mg} / \mathrm{kg} / \mathrm{day}(100 \mathrm{mg} /$ tablet: Radanil, Roche). During treatment, patients were subjected to clinical examination to evaluate side effects.

Blood samples were collected at the following instances: T0, before treatment; T1, two months; T2, three months; T3, six months; T4, one year; T5, two years and T6, three years after treatment onset, respectively.

The research protocol followed the tenets of the Declaration of Helsinki and was approved by the Local Medical Ethics Committees named Comité Institucional de Ética de Investigación en Salud - Ministerio de Salud y Desarrollo Social de Santiago del Estero and Comité de Ética del Hospital Zonal de Añatuya Monseñor Gottau. Before and during the beginning of this study, the area was under triatominae vector surveillance by official government sanitary agents and Mundo Sano Private Foundation (i.e., they undertook regular insecticidal actions against infestations). All enrolled adult patients gave written informed consent and the parent or guardian of the child participants provided informed consent on their behalf after the nature of the study was explained.

DNA extraction and amplification - Blood samples were mixed with an equal volume of $6 \mathrm{M}$ guanidine
HCl/0.2 M EDTA buffer pH: 8.0. Guanidine-EDTA blood (GEB) was heated for $15 \mathrm{~min}$ in boiling water and total DNA was purified from $500 \mu \mathrm{L}$ GEB with phenol-chloroform-isoamyl alcohol $(25: 24: 1, \mathrm{~V} / \mathrm{V})$ as previously reported (Schijman et al. 2003). The 330-bp variable regions of the T. cruzi kinetoplastid minicircle genome was amplified with 121 [5'-AAATAATGTACGG G(T/G)GAGATGCATGA-3'] and 122 (5'-GGTTCGATTGGGGTTGGTGTAATATA-3') primers by conventional PCR as previously described (Schijman et al. 2003).

Parasite lysate and recombinant proteins - Parasite extracts were obtained from T. cruzi epimastigotes CLBrener strain DTU Tc VI (Zingales et al. 2009), as previously described (Gómez et al. 2001).

B13, 1F8 and JL7 were expressed as GST fusion proteins and purified by affinity chromatography on glutathione-agarose beads as previously described (Umezawa et al. 1999). Protein contents were quantified by Bradford reagent, and all antigens were maintained at $-80^{\circ} \mathrm{C}$ until used.

Enzyme-linked immunosorbent assay (ELISA) - Inhouse ELISAs were carried out as described previously (Longhi et al. 2012). Briefly, microwells (Costar Inc., Corning, NY) were coated overnight at $4^{\circ} \mathrm{C}$ with $T$. cruzi lysate $(20 \mu \mathrm{g} / \mathrm{mL})$ or B13, $1 \mathrm{~F} 8$ and JL7 recombinant proteins $(5 \mu \mathrm{g} / \mathrm{mL})$ in $50 \mu \mathrm{L}$ of $0.05 \mathrm{M}$ carbonate-bicarbonate buffer ( $\mathrm{pH}$ 9.6). The plates were washed thrice with washing buffer (PBS containing $0.1 \%$ Tween-20; PBS-T) and then blocked with PBS-T plus 5\% non-fat dry milk for $1 \mathrm{~h}$ at $37^{\circ} \mathrm{C}$. Sera diluted 1:200 in PBS-T with $1 \%$ non-fat dry milk were incubated in duplicate for $2 \mathrm{~h}$ at $37^{\circ} \mathrm{C}$. To avoid test variability, serum samples (from T0T6) from a given patient were assayed on the same plate and sera from eight healthy individuals were also loaded on each plate to determine cut-off value.

After the plates were washed three times, $50 \mu \mathrm{L}$ of alkaline phosphatase (for T. cruzi lysate) or peroxidase (for recombinant proteins) conjugated anti-human polyvalent immunoglobulins diluted 1:3000 in PBS-T containing $1 \%$ non-fat dry milk, was dispensed into each well and incubated $1 \mathrm{~h}$ at $37^{\circ} \mathrm{C}$. The reaction was developed with p-nitrophenyl phosphate (for alkaline phosphatase) or 3, 3', 5, 5' - tetramethylbenzidine substrate (for peroxidase) and read at $415 \mathrm{~nm}$ or $450 \mathrm{~nm}$, respectively.

In parallel, some serum samples were also analysed by commercial Chagatest ELISA recombinant v4.0 (Wiener Laboratory, Rosario, Argentina) according to manufacturer's instruction.

Statistical analysis - Chi-square test for the analysis of drug side-effects were performed. Longitudinal panel data analysis was used to assess the change in Ab levels over time. Longitudinal data was analysed using generalised linear mixed effects modelling, as implemented in R software 2.10.0 version (R Core Team 2013), with random effects specified at the level of the individual. Normal distribution of dependent variables was evaluated with Shapiro-Wilks test. Variables not normally distributed were log-transformed before analysis and log-normality confirmed with Shapiro-Wilks test for the transformed variables. Estimation of $95 \%$ confidence intervals $(95 \%$ 


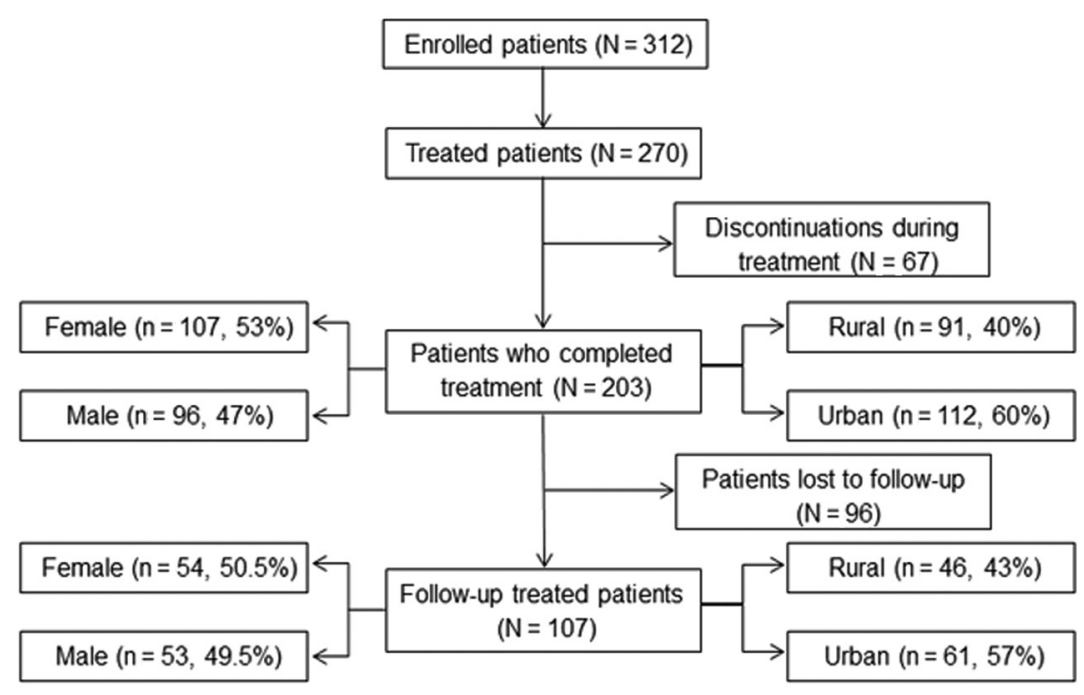

Fig. 1: flow diagram of patient enrollment, treatment and follow-up.

CI) for generalised linear mixed effects regressions was done by Markov Chain Monte Carlo, as implemented in WinBUGS and R (R Core Team 2013). Statistical significance was assumed at $\mathrm{p}<0.05$ or $95 \%$ CI.

\section{RESULTS AND DISCUSSION}

Several recombinant antigens are used for diagnosis of Chagas disease (Afranchino et al. 1989, dos Santos et al. 1992, Krieger et al. 1992, Umezawa et al. 1999, 2003, Di Noia et al. 2002, Camussone et al. 2009, De Marchi et al. 2011), but none of them has been tested as early markers of cure after anti-parasitic treatment. With this idea in mind, we decided to determine the level of antibodies against three antigens, namely B13, 1F8 and JL7, in a cohort of patients treated with BNZ by an in-house ELISA method. These antigens correspond to repeat tandem proteins of T. cruzi and have been well described in other works as immunodominant antigen in Chagas disease (Umezawa et al. 1999, 2003, Longhi et al. 2012).

Flowchart of enrollment, treatment and follow-up is schematised in Fig. 1. Sixty seven out of 270 enrolled patients $(24.8 \%)$ interrupted treatment, because of death $(2 / 67,2.9 \%)$, pregnancy $(4 / 67,5.9 \%)$, fail to correctly follow medical prescription or adverse effects $(24 / 67,35.8 \%)$ and voluntary abandonment (37/67, 55.2\%). Although 203 completed drug therapy, only three or more follow-up samples could be obtained in 107 treated patients, especially due to difficulties of patients to attend to hospital during three years of drug monitoring. The number of samples analysed from T0-T6 is shown in Table. It is important to mention that the group of patients analysed was representative of the total cohort that completed treatment with similar rates of females/males and rural/urban residents (Fig. 1). Moreover, mean age of patients who completed treatment (34.5 years, $\mathrm{N}=203$ ) or follow-up (35.7 years, $\mathrm{N}=107$ ) was also comparable, with a higher proportion of patients ranged in age from $25-44$ years $(65 \%$; $132 / 203$ and $68 \% ; 73 / 107$, respectively).
TABLE

Number of samples analysed during the benznidazole follow-up

\begin{tabular}{lcc}
\hline & Time $^{\mathrm{a}}$ & $\mathrm{N}^{\mathrm{b}}$ \\
\hline T0 & 0 & 107 \\
T1 & 2 & 104 \\
T2 & 3 & 106 \\
T3 & 6 & 104 \\
T4 & 12 & 73 \\
T5 & 24 & 65 \\
T6 & 36 & 60
\end{tabular}

${ }^{a}$ Time in months from treatment onset; ${ }^{b}$ Number of serum samples.

As it has been previously reported (Viotti et al. 2009, Pinazo et al. 2010) typical side effects, generally mild and transient, were observed in 67 out of 203 patients who complete treatment. The most common side effect proved cutaneous hypersensitivity $(29 / 203,14.3 \%)$ such as pruritus, allergy and exanthema, followed by digestive disorders (18/203, 8.8\%) as epigastric pain, dyspepsia and heartburn. In addition, 11 out of 203 patients (5.4\%) presented headache, nauseas or vomiting, while nine patients suffered arthralgia and myalgia (4.4\%). The appearance of more than one side effect was only observed in 12 out of 203 patients (5.9\%). The incidence of adverse reactions was not statistically associated to age, sex, or place of residence -urban or rural areas(Chi-square test, $\mathrm{p}=0.43, \mathrm{p}=0.26, \mathrm{p}=0.96$, respectively). It is important to highlight that a high number of patients, who developed cutaneous hypersensitivity, worked in brick factories exposed to heat and sunlight.

Parasitological experiments were performed in 74 out of 107 patients. At baseline, 60.8\% (45/74) had posi- 

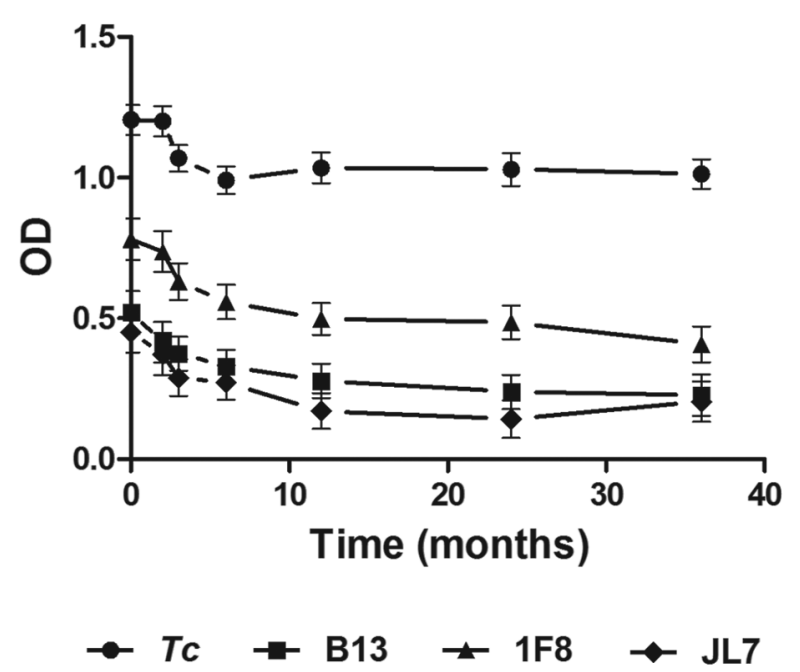

Fig. 2: antibody levels. ELISA plates were coated with Trypanosoma cru$z i$ lysate, or B13, 1F8 and JL7 recombinant proteins in buffer carbonate. Serum samples were diluted 1/200 and tested in duplicate. Results are expressed as optical density (OD) means for each antigen \pm standard error of the means (SEM). Cut-off values for T. cruzi, B13, 1F8 and JL7 recombinant proteins, were $0.276,0.110,0.367$ and 0.150 , respectively.

tive results for T. cruzi PCR, whereas during monitoring period, consistently negative PCR results were documented in 34/45 (75.5\%). Similar data have been reported by Morillo et al. (2015) in BENEFIT trial, showing that $T$. cruzi DNA was not detected in $73 \%$ of Argentinian and Bolivian patients at the end of follow-up. However, CHAGASAZOL trial reported that $94 \%$ of patients treated with BNZ tested negative by PCR (Molina et al. 2014). These differences could be related to the risk of exposure to the parasite, because patients included in CHAGASAZOL trial have been living in Barcelona, Spain - a non-endemic area - during the whole study. On the contrary, although the area was under triatominae vector surveillance, the study population in BENEFIT trial and in our work belonged to endemic regions, where there is a real risk of exposure to reinfections, due to population movement to areas without vector control.

After analysing serologic response against $T$. cruzi lysate or recombinant proteins by linear mixed effects modelling, we observed an apparent biphasic curve for all antigens, with an initial rapid decline within the first three months followed by a plateau with a slight decay for the remaining 36 months of follow-up (Fig. 2). The decrease in Ab titers against T. cruzi lysate over time was -0.0075 optical density (OD)/month (95\% CI -0.0060 to -0.0090$]$ ), with a significant effect of time for each patient (Supplementary figure). Similar results were obtained for $\mathrm{Ab}$ anti-B13, anti-1F8 and anti-JL7 levels with a slope of -0.0032 OD/month for B13 (95\% CI [-0.0010 to-0.0074], -0.0041 $\mathrm{OD} / \mathrm{month}$ for $1 \mathrm{~F} 8(95 \% \mathrm{CI}[-0.0001$ to -0.0083$])$ and $-0.0032 \mathrm{OD} / \mathrm{month}$ for JL7 (95\% CI [-0.0009 to -0.0075]), with significant effect for each patient as a function of time (Supplementary figure). A statistically significant faster rate of decline was observed for $\mathrm{Ab}$ titers against T. cruzi lysate in comparison to anti-B13, anti-1F8 and anti-JL7 ( $p<0.01$ for all comparisons, linear mixed effects model analysis). Indeed, results showed that the OD mean values for recombinant proteins declined progressively close to the cut-off values at the end of the followup, while those for T. cruzi lysate remained positive (Fig. 2 ). This observation could be explained by the fact that sera reactivity at $\mathrm{T} 0$ is higher to $T$. cruzi lysate than with recombinant proteins. This data is in accordance with results presented by others, who observed a low rate of seronegative conversion of anti-T. cruzi response detected by conventional serological test (Sosa-Estani et al. 1998, 2009, Viotti et al. 2011, Machado-de-Assis et al. 2013).

It is important to highlight that most sera presented reactivity against $T$. cruzi lysate and the recombinant proteins, as for example the Patient \#115 (Fig. 3), but some patients did not developed detectable $\mathrm{Ab}$ against all the recombinant antigens. As shown in Fig. 3, serum from Patient \#207 did not react with the three studied recombinant antigens and along with serum from Patient \#7 were the only two samples that showed low reactivity against B13. Likewise five patients had a similar pattern response as Patient \#131, seven patients as Patient \#85 and 12 patients as Patient \#27 without reactivity to $1 \mathrm{~F} 8$ and/or JL7 (Fig. 3). In this cohort of patients, the percentage of reactivity against B13, 1F8 and JL7 was 98, 89 and 82 , respectively. Based on these finding, we suggest that no single recombinant protein would be useful for the assessment of therapeutic response in all patients, and reactivity against the three antigens should be tested individually for future treatment evaluation.

In parallel for comparison purpose, sera reactivity was determined by a commercial Chagatest ELISA recombinant. As shown in Fig. 3, a similar pattern response to $T$. cruzi lysate was obtained, with a slight decrease in $\mathrm{Ab}$ levels, but without showing a seroconversion at end of follow-up, while as mentioned above, anti-B13, anti-1F8 and anti-JL7 Ab declined progressively close to the cutoff values at the end of follow-up. Finally, we observed along to monitoring that Patients \#115, \#131, \#7, \#85 and \#27 showed decay in Ab response, especially against to recombinant antigens (Fig. 3) and also tested negative for T. cruzi DNA by PCR. On the contrary, Patient \#207, who had no changes in reactivity against T. cruzi lysate (Fig. 3), PCR assays remained positive during the follow-up.

Throughout the years, there have been major advances in the control of $T$. cruzi transmission due to vector control by insecticide spraying, but little has been achieved in terms of treatment of infected people. The fact that most of those infected individuals are from the poorer strata of society diminishes the interest in the development of new drugs by the pharmaceutical companies, but it is one of the priorities for WHO in the control of Chagas disease. Therefore, therapy efficacy or drug success necessarily requires validate an accurate method to evaluate the course of the disease (Pinazo et al. 2014).

As mentioned above, positive prognosis after antiparasite treatment often is made by serological and/or parasitological subsequent examinations with consistently negative results in time (Porrás et al. 2015). Non- 
Patient \#115

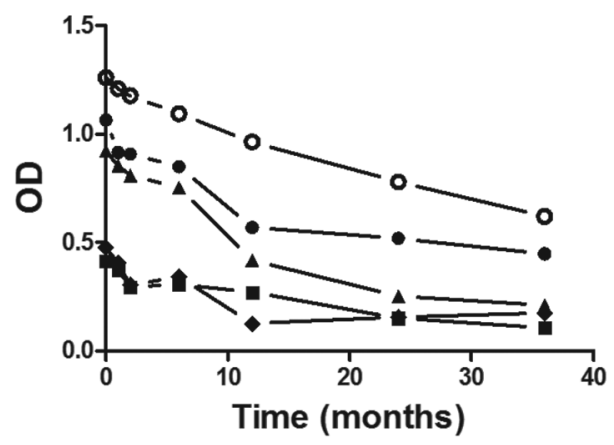

Patient \#131

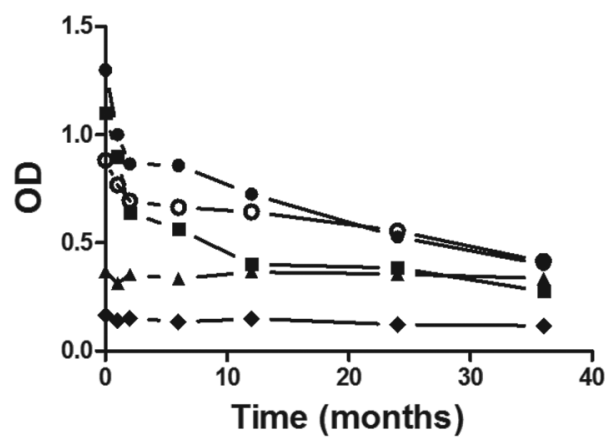

Patient \#85

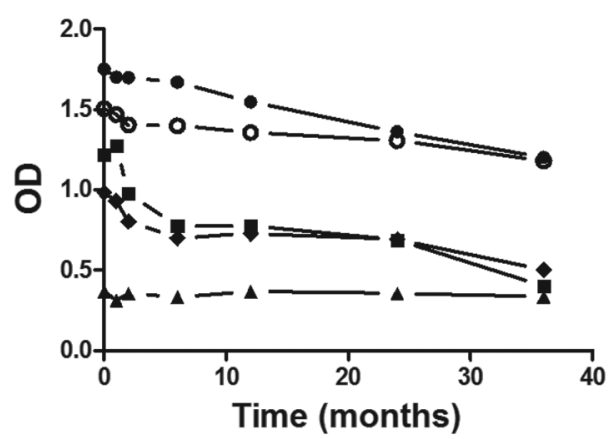

Patient \#207

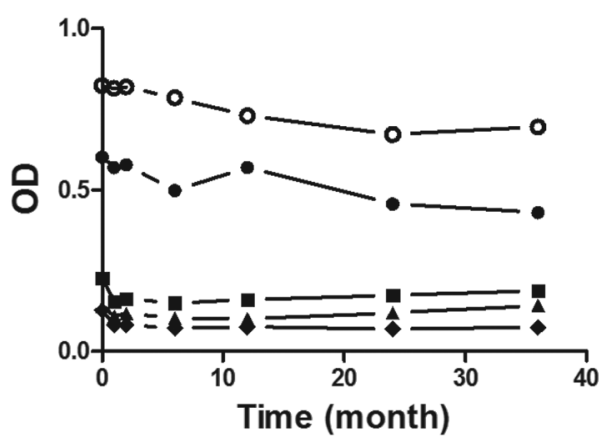

Patient \#7

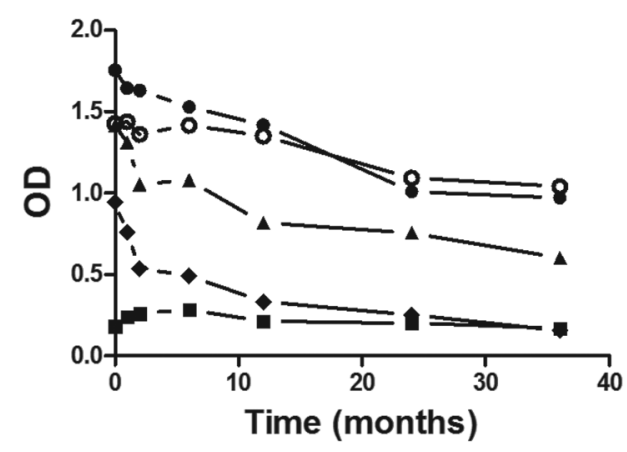

Patient \#27

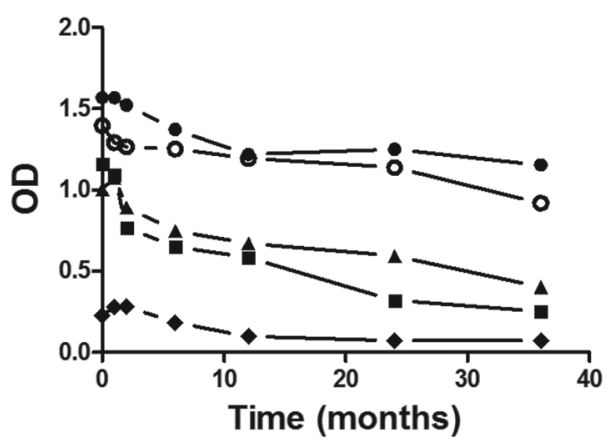

- Chagatest

Fig. 3: variation in optical density (OD) by the ELISA as a function of time. Results from six patients, representing typical pattern response against Trypanosoma cruzi lysate, B13, 1F8 and JL7 recombinant proteins, are schematised. Chagatest ELISA recombinant v4.0 results were included in comparison of in-house ELISAs. Cut-off values for T. cruzi, Chagatest, B13, $1 \mathrm{~F} 8$ and JL7 recombinant proteins, were 0.276, 0.126, 0.110, 0.367 and 0.150 , respectively.

conventional serological methods, including a multiplex format or ELISA with recombinant proteins, have been developed to provide fast, easy to measure, responses to etiological treatments (Cooley et al. 2008, FernándezVillegas et al. 2011, Fabbro et al. 2013). However, there are no consensuses about which is the gold standard of post-treatment evaluation protocols to establish cure in a reasonably short time (Urbina 2015).

Overall, our results showed that none of the patients showed a seronegative conversion for anti-T. cruzi response during the follow-up, but a significant decay in the $\mathrm{Ab}$ titer against recombinant antigens was observed 
in the majority of the patients with chronic Chagas disease three years after the end of specific drug treatment.

Since, the level of $\mathrm{Ab}$ against recombinant proteins B13, 1F8 and JL7 decreased to cut-off line earlier than Ab against $T$. cruzi lysate and these reduction were not statistically associated to age, sex, or place of residence, results suggest that these antigens would be good candidates as early markers of serological response to evaluate drug success. Although the main limitations of our study were the non-randomised design, and absence of a placebo control group due to bioethics considerations of the Local Medical Ethics Committee, our findings encourage further evaluation of these recombinant antigens in randomised and controlled trial with trypanocidal drugs.

\section{ACKNOWLEDGEMENTS}

To all patients who participated in this study, Añatuya community, the staffs of the Hospital Zonal Añatuya, Mundo Sano Foundation, for triatomine vector surveillance, and Ms Carina Romano, for technical assistance. We would like to dedicate this work to the memory of Dr Mariano J Levin and Ms Crescencia López.

\section{REFERENCES}

Affranchino JL, Ibanez CF, Luquetti AO, Rassi A, Reyes MB, Macina RA, et al. Identification of a Trypanosoma cruzi antigen that is shed during the acute phase of chagas' disease. Mol Biochem Parasitol. 1989; 34(3): 221-8.

Bianchi F, Cucunubá Z, Guhl F, González NL, Freilij H, Nicholls RS, et al. Follow-up of an asymptomatic Chagas disease population of children after treatment with nifurtimox (Lampit) in a sylvatic endemic transmission area of Colombia. PLoS Negl Trop Dis. 2015; 9(2): e0003465.

Camussone C, González V, Belluzo MS, Pujato N, Ribone ME, Lagier CM, et al. Comparison of recombinant Trypanosoma cruzi peptide mixtures versus multiepitope chimeric proteins as sensitizing antigens for imunodiagnosis. Clin Vaccine Immunol. 2009; 16(6): 899-905.

Cançado JR. Long term evaluation of etiological treatment of chagas disease with benznidazole. Rev Inst Med Trop Sao Paulo. 2002; 44(1): 29-37.

Cooley G, Etheridge RD, Boehlke C, Bundy B, Weatherly DB, Minning $\mathrm{T}$, et al. High throughput selection of effective serodiagnostics for Trypanosoma cruzi infection. PLoS Negl Trop Dis. 2008; 2(10): e316.

de Andrade AL, Zicker F, de Oliveira RM, Silva SA, Luquetti A, Travassos LR, et al. Randomised trial of efficacy of benznidazole in treatment of early Trypanosoma cruzi infection. Lancet. 1996; 348(9039): 1407-13.

De Marchi CR, Di Noia JM, Frasch AC, Amato Neto V, Almeida IC, Buscaglia CA. Evaluation of a recombinant Trypanosoma cruzi mucin-like antigen for serodiagnosis of chagas' disease. Clin Vaccine Immunol. 2011; 18(11): 1850-5.

Di Noia JM, Buscaglia CA, De Marchi CR, Almeida IC, Frasch AC. A Trypanosoma cruzi small surface molecule provides the first immunological evidence that chagas' disease is due to a single parasite lineage. J Exp Med. 2002; 195(4): 401-13.

dos Santos CN, Krieger MA, Almeida E, Lafaille JJ, Goldenberg S, Galler R. Trypanosoma cruzi flagellar repetitive antigen expression by recombinant baculovirus: towards an improved diagnostic reagent for chagas' disease. Biotechnology (NY). 1992; 10(11): 1474-7.

Fabbro D, Velazquez E, Bizai ML, Denner S, Olivera V, Arias E, et al. Evaluation of the ELISA-F29 test as an early marker of therapeu- tic efficacy in adults with chronic Chagas disease. Rev Inst Med Trop Sao Paulo. 2013; 55(3): 167-72.

Fernández-Villegas A, Pinazo MJ, Marañón C, Thomas MC, Posada E, Carrilero B, et al. Short-term follow-up of chagasic patients after benzonidazole treatment using multiple serological markers. BMC Infect Dis. 2011; 11: 206.

Gómez EB, Medina G, Ballesta JP, Levin MJ, Tellez-Iñón MT. Acidic ribosomal $\mathrm{P}$ proteins are phosphorylated in Trypanosoma cruzi. Int J Parasitol. 2001; 31(10): 1032-9.

Guedes PM, Silva GK, Gutierrez FR, Silva JS. Current status of Chagas disease chemotherapy. Expert Rev Anti Infect Ther. 2011; 9(5): 609-20.

Jackson Y, Gétaz L, Wolff H, Holst M, Mauris A, Tardin A, et al. Prevalence, clinical staging and risk for blood-borne transmission of Chagas disease among Latin American migrants in Geneva, Switzerland. PLoS Negl Trop Dis. 2010; 4(2): e592.

Krieger MA, Almeida E, Oelemann W, Lafaille JJ, Pereira JB, Krieger $\mathrm{H}$, et al. Use of recombinant antigens for the accurate immunodiagnosis of chagas' disease. Am J Trop Med Hyg. 1992; 46(4): 427-34.

Lescure FX, Canestri A, Melliez H, Jauréguiberry S, Develoux M, Dorent R, et al. Chagas disease, France. Emerg Infect Dis. 2008; 14(4): 644-6.

Longhi SA, Brandariz SB, Lafon SO, Niborski LL, Luquetti AO, Schijman AG, et al. Evaluation of in-house ELISA using Trypanosoma cruzi lysate and recombinant antigens for diagnosis of Chagas disease and discrimination of its clinical forms. Am J Trop Med Hyg. 2012; 87(2): 267-71.

Machado-de-Assis GF, Diniz GA, Montoya RA, Dias JCP, Coura JR, Machado-Coelho GLL, et al. A serological, parasitological and clinical evaluation of untreated Chagas disease patients and those treated with benznidazole before and thirteen years after intervention. Mem Inst Oswaldo Cruz. 2013; 108(7): 873-80.

Machado-de-Assis GF, Silva AR, do Bem VA, Bahia MT, MartinsFilho OA, Dias JCP, et al. Posttherapeutic cure criteria in Chagas' disease: conventional serology followed by supplementary serological, parasitological, and molecular tests. Clin Vaccine Immunol. 2012; 19(8): 1283-91.

Molina I, Gómez i Prat J, Salvador F, Treviño B, Sulleiro E, Serre $\mathrm{N}$, et al. Randomized trial of posaconazole and benznidazole for chronic Chagas' disease. N Engl J Med. 2014; 370(20): 1899-908.

Morillo CA, Marin-Neto JA, Avezum A, Sosa-Estani S, Rassi Jr A, Rosas F, et al. Randomized trial of benznidazole for chronic Chagas' cardiomyopathy. N Engl J Med. 2015; 373(14): 1295-306.

Muñoz J, Gomez i Prat J, Gallego M, Gimeno F, Treviño B, LópezChejade P, et al. Clinical profile of Trypanosoma cruzi infection in a nonendemic setting: immigration and Chagas disease in Barcelona (Spain). Acta Trop. 2009; 111(1): 51-5.

PAHO - Pan American Health Organization. Etiological treatment of Chagas disease. Conclusions of a technical consultation. Washington DC: OPS/HCP/HCT/140/99; 1999.

Pinazo MJ, Muñoz J, Posada E, López-Chejade P, Gállego M, Ayala E, et al. Tolerance of benznidazole in treatment of Chagas' disease in adults. Antimicrob Agents Chemother. 2010; 54(11): 4896-9.

Pinazo MJ, Thomas MC, Bua J, Perrone A, Schijman AG, Viotti RJ, et al. Biological markers for evaluating therapeutic efficacy in Chagas disease, a systematic review. Expert Rev Anti Infect Ther. 2014; 12(4): 479-96.

Ponce C, Ponce E, Vinelli E, Montoya A, de Aguilar V, Gonzalez A, et al. Validation of a rapid and reliable test for diagnosis of chagas' disease by detection of Trypanosoma cruzi-specific antibodies in blood of donors and patients in Central America. J Clin Microbiol. 2005; 43(10): 5065-8. 
Porrás AI, Yadon ZE, Altcheh J, Britto C, Chaves GC, Flevaud L, et al. Target product profile (TPP) for Chagas disease point-of-care diagnosis and assessment of response to treatment. PLoS Negl Trop Dis. 2015; 9(6): e0003697.

R Core Team. R Core Team R: a language and environment for statistical computing [Internet]. Vienna: R Foundation for Statistical Computing; 2013. Available from: http://www.R-project.org/.

Riarte A. TRAENA: Placebo-controlled evaluation of impact of benznidazole treatment on long-term disease progression in adults with chronic Chagas disease. In: Proceedings of the 62nd Annual Meeting of the American Society of Tropical Medicine and Hygiene; November 13-17, 2013; Washington DC.

Schijman AG, Altcheh J, Burgos JM, Biancardi M, Bisio M, Levin MJ, et al. Aetiological treatment of congenital Chagas' disease diagnosed and monitored by the polymerase chain reaction. J Antimicrob Chemother. 2003; 52(3): 441-9.

Sosa-Estani S, Segura EL, Ruiz AM, Velazquez E, Porcel BM, Yampotis $\mathrm{C}$. Efficacy of chemotherapy with benznidazole in children in the indeterminate phase of Chagas' disease. Am J Trop Med Hyg. 1998; 59(4): 526-9.

Sosa-Estani S, Viotti R, Segura EL. Therapy, diagnosis and prognosis of chronic Chagas disease: insight gained in Argentina. Mem Inst Oswaldo Cruz. 2009; 104(Suppl. 1): 167-80.

Umezawa ES, Bastos SF, Camargo ME, Yamauchi LM, Santos MR, Gonzalez A, et al. Evaluation of recombinant antigens for serodiagnosis of Chagas' disease in South and Central America. J Clin Microbiol. 1999; 37(5): 1554-60.
Umezawa ES, Bastos SF, Coura JR, Levin MJ, Gonzalez A, Rangel-Aldao R, et al. An improved serodiagnostic test for Chagas' disease employing a mixture of Trypanosoma cruzi recombinant antigens. Transfusion. 2003; 43(1): 91-7.

Urbina JA. Recent clinical trials for the etiological treatment of chronic Chagas disease: advances, challenges and perspectives. J Eukaryot Microbiol. 2015; 62(1): 149-56.

Viotti R, Alarcón de Noya B, Araujo-Jorge T, Grijalva MJ, Guhl F, López $\mathrm{MC}$, et al. Towards a paradigm shift in the treatment of chronic Chagas disease. Antimicrob Agents Chemother. 2014; 58(2): 635-9.

Viotti R, Vigliano C, Alvarez MG, Lococo B, Petti M, Bertocchi G, et al. Impact of aetiological treatment on conventional and multiplex serology in chronic Chagas disease. PLoS Negl Trop Dis. 2011; 5(9): e1314.

Viotti R, Vigliano C, Lococo B, Alvarez MG, Petti M, Bertocchi G, et al Side effects of benznidazole as treatment in chronic Chagas disease: fears and realities. Expert Rev Anti Infect Ther. 2009; 7(2): 157-63.

Viotti R, Vigliano C, Lococo B, Bertocchi G, Petti M, Alvarez MG, et al. Long-term cardiac outcomes of treating chronic Chagas disease with benznidazole versus no treatment: a nonrandomized trial. Ann Intern Med. 2006; 144(10): 724-34.

WHO - World Health Organization. Chagas disease in Latin America: an epidemiological update based on 2010 estimates [Internet]. Geneva: WHO; 2015. Available from: http://www.who.int/ wer/2015/wer9006.pdf?ua $=1$.

Zingales B, Andrade SG, Briones MRS, Campbell DA, Chiari E, Fernandes $\mathrm{O}$, et al. A new consensus for Trypanosoma cruzi intraspecific nomenclature: second revision meeting recommends TcI to TcVI. Mem Inst Oswaldo Cruz. 2009; 104(7): 1051-4. 


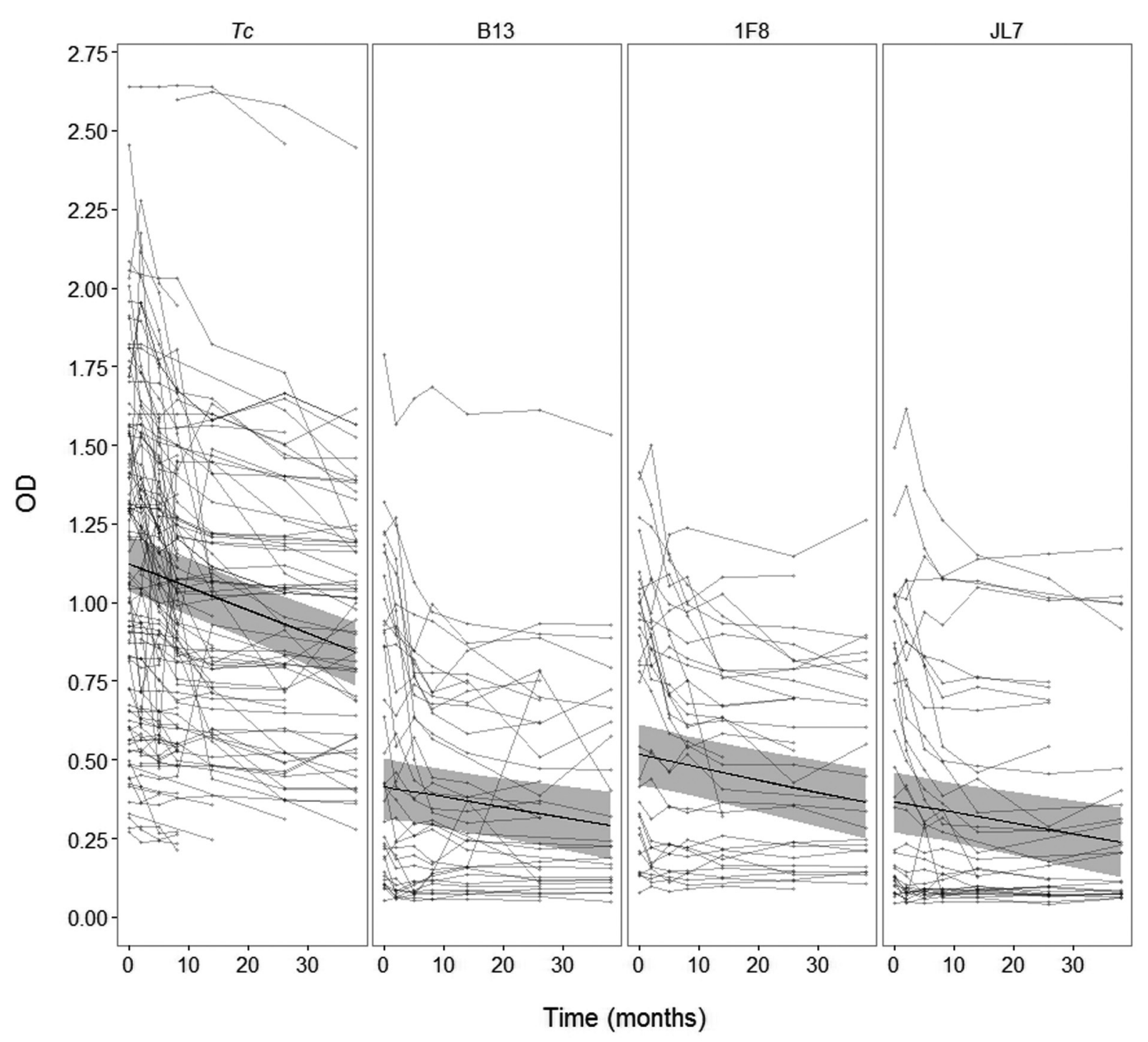

Linear mixed effects modelling. ELISA plates were coated with Trypanosoma cruzi lysate, or B13, 1F8 and JL7 recombinant proteins in buffer carbonate. Serum samples were diluted 1/200 and tested in duplicate. Each point corresponds to optical density (OD) mean value for each patient as function of time. Bold lines represent linear mixed effects regression, and grey zone $95 \%$ confidence intervals. Cut-off values for $T$. cruzi, $\mathrm{B} 13,1 \mathrm{~F} 8$ and JL7 recombinant proteins, were $0.276,0.110,0.367$ and 0.150 , respectively. 